\title{
A New Double Probe System for Studies of Non-Uniform Plasmas
}

\author{
G. Petraconi and H. S. Maciel \\ Instituto Tecnológico de Aeronáutica- ITA, Laboratório de Plasma e Processos- LPP \\ Praça Marechal Eduardo Gomes, 50, 12228-900, São José dos Campos, SP, Brazil
}

Received on 25 February, 2003. Revised version received on 22 May, 2003

\begin{abstract}
A theoretical and experimental study was developed about the applicability of a double probe system consisting of two directional Langmuir probes, both probes being located separately in a plasma column. The current- voltage characteristic of the double probe was obtained considering a plasma with a drifting maxwellian electron velocity distribution function and stationary ion background. In deriving the characteristic of the double probe, the plasma parameters, namely, electron temperature $\left(T_{e}\right)$, electron density $\left(N_{e}\right)$, electron drift velocity $\left(V_{d e}\right)$ and plasma potential $\left(V_{p}\right)$ are assumed to be non-uniform. The double probe characteristic is also dependent on the angle between the axial direction of the electron drift and the normal to the collecting area of the probe. Each probe can be rotated such that this angle can be varied between zero and 180 degrees. Various probe characteristics were simulated using plasma parameters obtained by independent single probe measurements in the positive column of a low-pressure arc discharge in mercury vapor. Typical parameters of the positive column, used in the simulation, are: $T_{e}=5 \mathrm{eV}, N_{e}=10^{17} \mathrm{~m}^{-3}, v_{d e}=8 x 10^{5} \mathrm{~ms}^{-1}$. Experimental characteristics of the double probe were obtained and compared with the simulated results, showing good agreement. It is concluded that this directional probe system can be a reliable diagnostic tool especially for studies of non-uniform plasmas.
\end{abstract}

\section{Introduction}

Double probe method [1,2] is widely used for the study of plasmas properties. Most of the probe theories consider the case of a plasma at rest. However, in some plasmas $[3,4]$ the electron drift velocity in the axial direction, which is principally responsible for carrying the discharge current, may reach an appreciable fraction of the thermal velocity, altering the electron drift velocity distribution from maxwellian to Drift-maxwellian form. Under these circumstances, it is important to recognize the signature of the drifting electrons in the double probe characteristic. Ariga [5] has theoretically treated the characteristic of double probes immersed in plasma in which electrons and ions have a drift velocity in the axial direction, with the ion saturation current dependent on the ion temperature $\left(T_{i}\right)$.

In this work, we consider the case of a plasma column of a low-pressure mercury arc dishcarge and we assume that the velocity distribution function of the electrons at the axis is maxwellian, with a superposed drift in the axial direction. The ion current collected by the probe is given by the Bohm's current [1]. Thus, the ion flux is assumed without drift and not affected by the probe potential. The basic equations for the electron and ion currents are shown in section 2 , including the analysis of the floating potential and of the slope of the double probe characteristic near zero current. The effect of electron drift velocity on the double probe characteristic was simulated inserting the plasma parameters determined along the axis of the plasma column using single electrostatic probes. In section 3 , we provide a concrete example of the application of this model by fitting data from a low-pressure mercury-arc discharge [3,4]. Agreement with the theoretical model is found quite good.

\section{Directional double probe method}

Directional double probe system is constituted by two plane Langmuir probes introduced into the plasma and connected to a dc symmetric supply unit so as to vary not only the voltage $V_{a}$ between the probes but its polarity as well. Fig. 1 shows the double probe measuring circuit and the potential distribution in a floating double probe system. In this figure, $V_{a}$ is the differential voltage applied between the probes, is the potential difference between the regions of the plasma where the probes are located $\left(V_{p 2}--V_{p 1}\right), V_{1}$ and $V_{2}$ are the potential difference between the probe and the surrounding plasma.

Each probe can be rotated such that the angle $(\theta)$ between the axial direction of the electron drift and the normal to the collecting area of the probe can be varied between zero and 180 degrees. In this work, unlike the case of an ordinary double probe system, the distance (s) between the probes can be varied at large values. $V_{f 1}$ and $V_{f 2}$ are the floating potential of the probes 1 and 2 when the angle $\theta$ of both probes is $0^{0}$ (frontal electrodes). The floating double probe circuit having back to back electrodes is obtained by rotation of the probe 2 at the angle $\theta$ of $180^{\circ}$, in this case, the floating potential of the probe 2 is $V_{f 2}^{\prime}$.

\subsection{Derivation of the double probe character- istic equation}

In this analysis, we consider the electron and ion current collected by a double planar probe in non-uniform plasma with 
drifting electrons. The $z$-axis is perpendicular to the probe surface and points to it. That is, electrons with a $z$

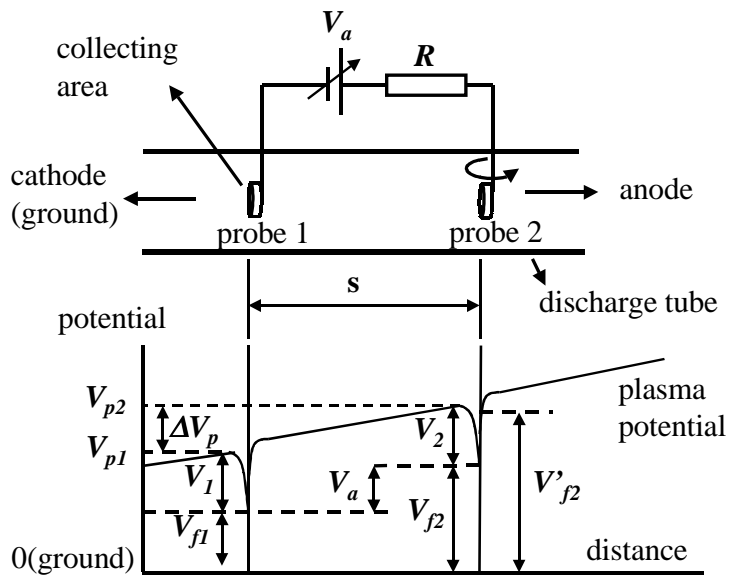

Figure 1. Diagram of the circuit for operating a floating double probe and the potential distribution in a floating double probe system.

component of velocity $\nu_{z}>0$ are moving toward the probe surface, and those with $\nu_{z}<0$ are moving away. The probe is immersed in a plasma where the electron distribution function is given by:

$$
f(\nu)=N_{e}\left(\frac{m}{2 \pi k_{b} T_{e}}\right)^{1 / 2} \exp \left[-\frac{m}{2 k_{B} t_{e}}\left(\nu-\nu_{d}\right)^{2}\right]
$$

Where $N_{e}, T_{e}, \nu_{d}$ and $m$ are the density, temperature, drift velocity and mass of the electrons respectively, $\nu=\nu_{z}$ is the axial velocity, and $k_{B}$ is the Boltzmann's constant.

The electron current collected at a single directional probe surface as a function of the retarding bias can be found as follows. The probe will collect only those electrons with . Here $e$ is the electron charge, and $V$ is the probe potential taken with respect to the plasma potential $V_{P}$. Thus the electron current at the two probes is given by [3]:

$$
I_{e 1}\left(\theta_{1}, V_{1}\right)=\left\{\exp \left[-U_{e 1}^{2}\right]+\frac{R_{1} \cos \theta_{1}}{\sqrt{2}} \operatorname{erfc}\left[U_{e 1}^{2}\right\} i_{e o 1}\right.
$$

and

$$
I_{e 2}\left(\theta_{2}, V_{2}\right)=\left\{\exp \left[-U_{e 2}^{2}\right]+\frac{R_{2} \cos \theta_{2}}{\sqrt{2}} \operatorname{erfc}\left[U_{e 2}^{2}\right\} i_{e o 2}\right.
$$

where

$$
\begin{aligned}
& U_{e 1}=\sqrt{\frac{V_{1}}{T_{e 1}}}-\frac{R_{1} \cos \theta_{1}}{\sqrt{2}}, \\
& U_{e 2}=\sqrt{\frac{V_{2}}{T_{e 2}}}-\frac{R_{2} \cos \theta_{2}}{\sqrt{2}},
\end{aligned}
$$

and

$$
\begin{aligned}
& i_{e o 1}=A_{1} N_{e 1} e \sqrt{\frac{e T_{e 1}}{2 \pi m_{e}}}, \\
& i_{e o 2}=A_{2} N_{e 2} e \sqrt{\frac{e T_{e 2}}{2 \pi m_{e}}},
\end{aligned}
$$

Here $i_{e o}$ is the random electron current in the axial direction, $A$ is the area of the probe, $T_{e}$ is the electron temperature in electron volts, $R$ is the ratio of the drift velocity $v_{d}$ to the electron thermal velocity and erfc $(x)$ is the complementary error function. Thus the current $I(\theta, V)$ has both exponential and error function dependencies on the retarding bias and depend only on the component of the drift velocity perpendicular to the probe surface $v_{d} \cos \theta$. Consequently, for $\theta=90^{\circ}$, the electron drift plays no role on the probe characteristic. Finally, $V_{1}$ and $V_{2}$ are related by the equation (see Fig. 1):

$$
V_{a}-\Delta V_{P}=V_{1}-V_{2}
$$

Besides, as the ions are assumed not to be affected by the probe potential the ion current $\left(I_{i}\right)$ flowing into the two plane probes of areas $A_{1}$ and $A_{2}$ are [3]

$$
I_{i 1} \approx 0,4 A_{1} e N_{i 1} \sqrt{\frac{e T_{e 1}}{m_{i}}}
$$

and

$$
I_{i 2} \approx 0,4 A_{2} e N_{i 2} \sqrt{\frac{e T_{e 2}}{m_{i}}}
$$

The effect of ion drift and electron drift velocities on the ion collection, more precisely in the Bohm's speed, which may occur at the transitional regions of the sheath is neglected for simplicity, though it may play an important role in the formation of the ion saturation current.

The current flowing in the external circuit is given by:

$$
I_{d p}=I_{i 1}+I_{e 1}=-\left(I_{e 2}+I_{i 2}\right.
$$

Equations (2) to (11) give the expression for the double probe characteristic:

$$
I_{d p}=\left[\frac{I_{i 2}(B-1)}{B C+1}\right]
$$

where

$$
B=\frac{\exp \left[-U_{e 2}^{2}+\frac{R_{2} \cos \theta_{2}}{\sqrt{2}} \operatorname{erfc}\left[U_{e 2}\right]\right.}{\exp \left[-U_{e 1}^{2}+\frac{R_{1} \cos \theta_{1}}{\sqrt{2}} \operatorname{erfc}\left[U_{e 1}\right]\right.}
$$

and

$$
C=\frac{N_{e 2} A_{2}}{N_{e 1} A_{1}} \sqrt{\frac{T_{e 2}}{T_{e 1}}}
$$

If $C=1$ (uniform plasma) and $R=0$ (no drift) a simple equation for the ordinary double probe characteristic is obtained [1]:

$$
I_{d p}=I_{i} \tanh \left[\frac{\Delta V_{P}-V_{a}}{2 T_{e}}\right]
$$


This expression indicates that the characteristic should be symmetrical with respect to $V_{a}=\Delta V_{P}$. In this case, the plasma potentials at the points where the probes are placed, $V_{P 2}$ and $V_{P 1}$, are different. For both probe to float there must be at a potential difference $V_{a}=\Delta V_{P}$ between them. By varying the voltage to bring the probe current to zero, and knowing the distance $\mathrm{s}$ between the probes, we estimate the axial electric field $E_{Z}=\Delta V_{P} / s$. Here, $\Delta V_{P}$ has the same meaning of the floating potential of the double probe.

The situation which that the plasma parameters are identical in the positions of the probes can be reproduced by positioning the probes side by side $(s=0)$. Fig. 2 shows the effect of the electron drift velocity on theoretical probe characteristic [eq. 12] for a double probe having two frontal electrodes when the distance between the probes (s) is zero(uniform plasma). For these fits the electron density used was $N_{e}=10^{17} \mathrm{~m}^{-3}$ and the electron temperature was $T_{e}=5 \mathrm{eV}$. If the plasma is uniform and if the plasma potentials $V_{P}$ at the two probes are equals, then no current flows though the probe circuit at zero voltage $\left(V_{a}=0, I_{d P}=0\right)$, because in this case the two probes are at the same floating potential.



Figure 2. Theoretical characteristic [eq. 12] of the double probe having two frontal electrodes in an uniform plasma. In order to evaluate only the electron drift velocity effect on each fit, all the other parameters involved in this simulation were kept fixed.

The double probe characteristic shown in Fig. 2 clearly shows that the electron drift velocity changes slightly the inclination of the characteristic curve. The double probe characteristic for non-uniform plasma is more complicated to obtain by simulation, because it involves the variation of many parameters. This part is discussed in the section 3 where probe data from a mercury arc discharge [6] are fitted for comparison with this theoretical model.

\subsection{Floating potential}

The floating potential of the double probe for a non-uniform plasma with drifting electrons, as considered here, is a function of the angle $\theta$, of the plasma potential $V_{P}$, of the electron drift velocity $v_{d}$ and electron temperature $T_{e}$ in each position of the probes. As the model treated here is nonlinear, this function was evaluated in the limit when $B \rightarrow 1$ in eq. (12). The following equation is a good approximation to $V_{f}$ when $R_{1} \cos \theta_{1} \rightarrow R_{2} \cos \theta_{2}$ because under this conditions the dependency of the floating potential with the electron drift velocity is weak:

$$
\begin{aligned}
V_{f}=\Delta V_{P} & -V_{1}\left(\frac{T_{e 2}-T_{e 1}}{T_{e 1}}\right)+T_{02}\left(R_{1} \cos \theta_{1}-R_{2} \cos \theta_{2}\right) \\
\times & {\left[\sqrt{\frac{2 V_{1}}{T_{e 1}}}-\frac{\left(R_{1} \cos \theta_{1}-R_{2} \cos \theta_{2}\right)}{2}\right] }
\end{aligned}
$$

Thus, if the plasma is non-uniform and if it transports drifting electrons, to attain the floating potential of the double probe it is necessary to overcome, by the increase of the bias voltage of the probe not only the difference of plasma potential, but also, the potential difference due to the variation of the electron temperature and also due to the difference of the electron drift velocity in the positions of the probes.

If $R_{1} \cos \theta_{1}=R_{2} \cos \theta_{2}$ or if the angles $\theta$ are $90^{\circ}$ in eq. (16), we obtain a simple expression to the floating potential of the double probe:

$$
V_{f}=\Delta V_{P}-\alpha T_{e}
$$

Here $V_{f}=V_{f 2}--V_{f 1}, \Delta T_{e}=T_{e 2}-T_{e 1}$ and $\alpha=V_{1} / T_{e 1}$ accounts for the drop in potential across the probe sheath. According to the mercury positive-column theory, if the electrons and ions have both ideal maxwellian distribution functions, the expected values of $\alpha$ is about 5.5. Under the existence of the drifting electrons, however, the $\alpha$ parameter should be evaluated by including the effect of the drift velocity on the Bohm's speed. As the equation (17) no has direct dependency with the electron drift velocity, it also can be obtained by analyzing the equation (8). Under these conditions, $I_{d P}=0$ because the drops in potential across the sheath of the probe $1\left(V_{1} / T_{e 1}\right)$ is equal to that of the probe $2\left(V_{2} / T_{e 2}\right)$ and the probes collect the same amount of ions and electrons current.

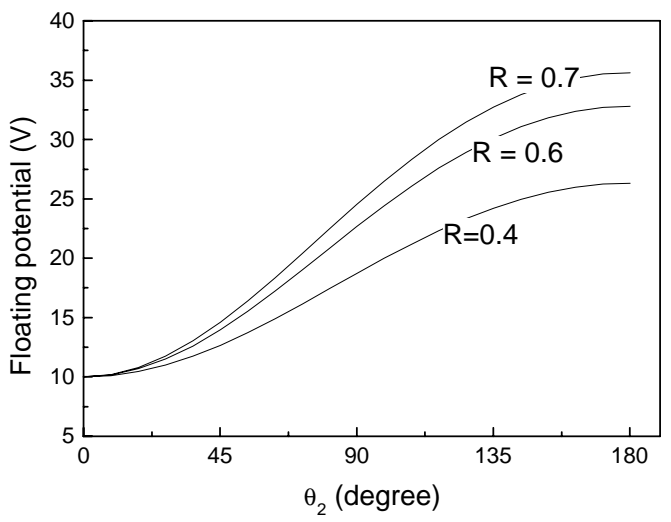

Figure 3. Effect of the electron drift velocity and of the angle $\theta_{2}$ on the floating potential. In order to evaluate the $R$ effect on each fit, all the other parameters involved in the equation (16) were kept fixed. The curves were evaluated assuming $R_{2}=R_{1}$ and $T_{e 2}=T_{e 1}$. The plasma parameters used for making this simulation were: $T_{e 1}=T_{e 2}=7.0 \mathrm{eV}, N_{1}=N_{2}=2.0 \times 10^{17} \mathrm{~m}^{-3}$, $\Delta V_{p}=10 \mathrm{~V}$ and $\alpha=5.5$. 
The effects of the electron drift velocity on the floating potential according the equation (16) is shown in the Fig. 3. The probe 1 was fixed at angle $\theta_{1}=0^{\circ}$ and the angle $\theta_{2}$ was varied between zero and 180 degrees.

The floating potential is minimum with respect to the plasma potential when the collecting area of the probe 2 faces away the electron drift velocity $\left(\theta_{2}=0\right)$. Fig. 3 clearly shows that the range of variation of the floating potential becomes larger by increasing the electron drift velocity. Considering for the analysis of a single curve ( $R$ is kept constant), that the electron axial velocity distribution function is not symmetrical, the key point that must be recognized is that a large bias must be applied to repel the drifting electrons that contribute to the $+R$ component (positive tail of maxwellian) of the double probe current, corresponding to the electron current flowing towards probe 1 . Contrary effect occurs with respect the probe 2 when the angle $\theta_{2}$ approaches $180^{\circ}$. In this case the contribution of the electrons of the $-R$ component (negative tail of the maxellian) of the double probe current, becomes lower and for the double probe to float (zero current), it needs to collect a lower ion flux, consequently the floating potential of the double probe approaches that of the plasma.

Although extrapolating the limits imposed by the model in computing these fits, the Fig. 3 shows that the results are consistent with works published by other authors $[3,5]$. In practice, however, effects of deformation of the plasma sheath and of drifting ions can generate significant discrepancies with respect to the theoretical model, mainly for angles $\theta$ greater than 90 degrees. These discrepancies are discussed in 3.1 .

\subsection{Slope analysis}

The probe theory suggests that the electron temperature and electron drift velocity can be estimated from the slope of the double probe characteristic at $V_{f}$, because the slope of the characteristic curve is affected by $T_{e}$ and $R$ [5]. Differentiating the equation (12) at $V_{a}=V_{f}$ and by expanding the complementary error function in an asymptotic series under the condition $R_{1} \cos \theta_{1}=R_{2} \cos \theta_{2}$, we obtain a good approximation for the slope of the double probe characteristic in a non-uniform plasma having drifting electrons:

$$
\left[\frac{d I_{d p}}{d V}\right]_{V_{a}=V_{f}}=\frac{I_{i 1} I_{i 2}}{\left(I_{i 1}+I_{i 2}\right) T_{e 2}}\left(\sqrt{\frac{1}{2 \alpha}} R \cos \theta-1\right)
$$

Note that, if $R=0$ or if $\theta=90^{\circ}$, the slope has the already known dependency on the electron temperature for a non-uniform plasma [1],

$$
\left[\frac{d I_{d p}}{d V}\right]_{V_{a}=V_{f}}=-\frac{I_{i 1} I_{i 2}}{\left(I_{i 1}+I_{i 2}\right) T_{e 2}} .
$$

Figure 4 shows the effect of the electron drift velocity on the slope of the double probe characteristics computed by means of the equation (18) for frontal probes.

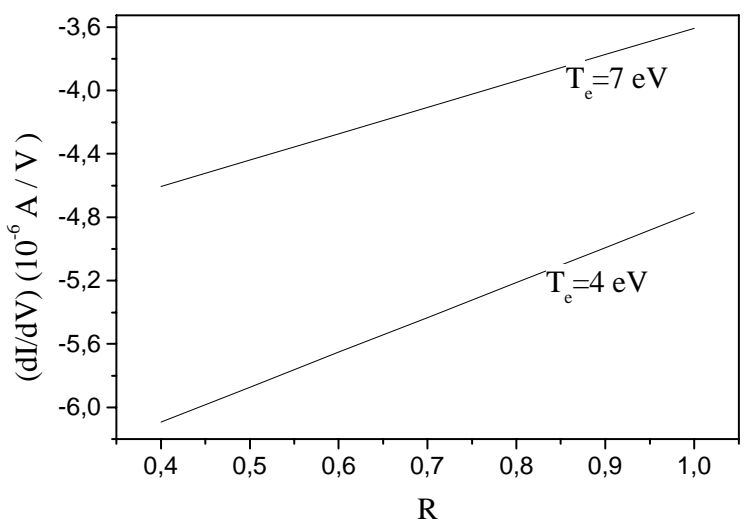

Figure 4. Effect of the electron drift velocity on the slope of the double probe characteristic for frontal probes configuration. In order to evaluate the $R$ effect on each theoretical fit, $T_{e}$ is kept constant.

As the system float, the probe potential is always negative with respect to the plasma potential. Consequently, the probe will collect only the electrons of the tail of the maxwellian. As result, the variation of the slope of the characteristic curve due to the effect of the $R$ is small when compared with the effect due to the electron temperature. However, as Fig. 4 shows, the drift changes almost linearly the slope of the characteristic curve; the larger the drift velocity the gentler becomes the slope of the double probe, in the case of frontal probes.

\section{Comparison with experiment}

The experiments were performed in a discharge system similar to that of Maciel and Allen [3] (Fig. 5), where the concept of a single directional probe was reported. The discharge current and the distance between the probe positions in the plasma column were kept constant at $2 \mathrm{~A}$ and $0.51 \mathrm{~m}$, respectively. The collecting area of the probes used were: $A_{1}=4.2 \times 10^{-6} \mathrm{~m}^{2}$ and $A_{2}=3.4 \times 10^{-6} \mathrm{~m}^{2}$.

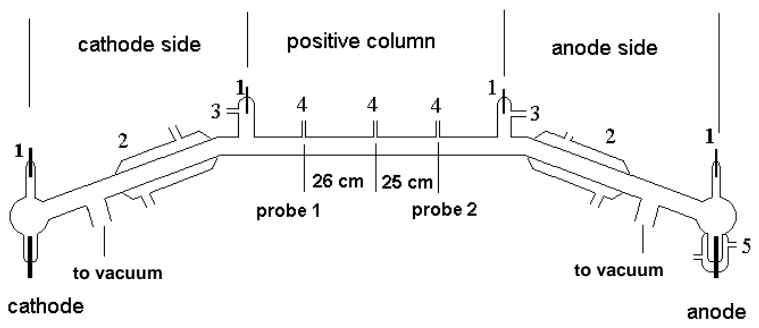

Figure 5. Experimental Apparatus. 1 - auxiliary electrode. 2 cooling Jacket. 3 - ionization (pressure) gauge. 4 - fixed probe in the positions 1 and 2. 5. - cooling jacket surrounding the mercury pool. 


\subsection{Floating potential}

Figure 6 shows the variation of the experimental values (solid line) of the floating potential with the angle $\theta_{2}$ when the angle $\theta_{1}$ is kept fixed at $0^{\circ}$. For comparison, the fit to the drifting electron model obtained by solving the equation (16) is indicated with dashed line.

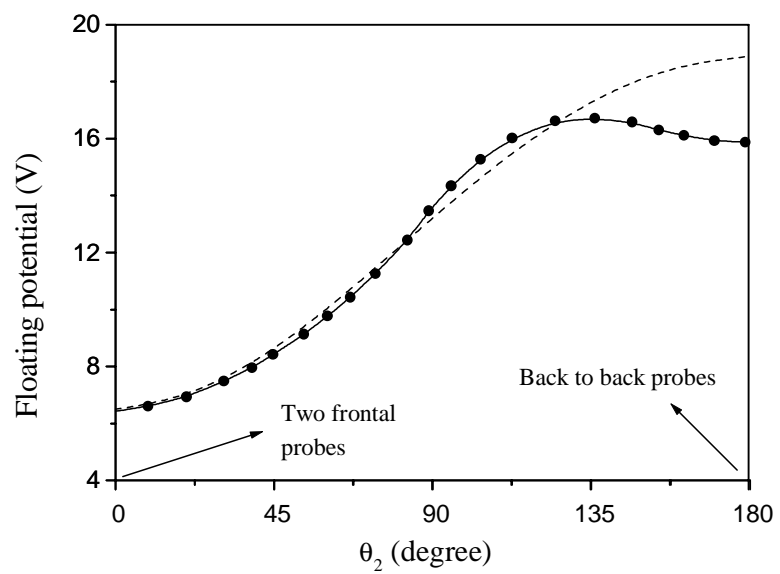

Figure 6. Comparison between the variation of the floating potential with the angle $\theta_{2}$, predicted from the drifting electron model (eq.16) and the floating potential measured in a mercury arc discharge [6]. Experimental data are indicated with solid line and the fitting curve by the dashed line. For this fit the plasma parameters used according to experimental results were: $R_{1}=0.52$, $R_{2}=0.50, T_{e 1}=5.4 \mathrm{eV}, T_{e 2}=4.4 \mathrm{eV}, N_{1}=1.85 \times 10^{17} \mathrm{~m}^{-3}$, $N_{2}=3.40 \times 10^{17} \mathrm{~m}^{-3}, \Delta V_{p}=1 \mathrm{~V}$.

The result of Fig. 6 shows that for angles $\theta$ highest than $130^{\circ}$ a disagreement between the two curves becomes evident by reason of the electrons of the negative tail of the maxwellian which are collected in a amount greater than that estimated from the eq. (16). This effect implies an increase of the flotation potential with respect to the plasma potential. Note in particular that the floating potential is significantly overestimated by the theoretical model for the case of back to back probes. Deformation of the probe sheath, considered to be plane in our model, is no longer valid in this range of angle $\theta_{2}$. Additionally, as the configuration approaches that of back to back probes the effect of the ion drift velocity towards probe 2 is also conceivable to contribute to the discrepancies between the curves of Fig. 6 . This observation suggests that an improvement of the model could be achieved by considering, in the theory, the effect of the ion and electron drift velocities on the Bohm's speed when $T_{e}<<T_{i}$. Thus, for the analysis presented in the next section, we have restricted to the case of the frontal probes configuration, where the effects explained above is not important because the probes receive equal ion saturation current.

\subsection{Double probe characteristics for the frontal probe configuration}

Figure 7(a) shows the experimental characteristic of the double probe made of two frontal probes. For comparison, the theoretical curves are shown in figure 7(b). In these curves, the plasma parameters were adjusted by a fitting method to give the same floating potential and slope of the experimental double probe characteristic. An interactive method based on the general double probe characteristic equation (eq. 12) was performed leading to the determination of the remaining plasma parameters comprised in the theoretical probe characteristic equation.

(7 a)

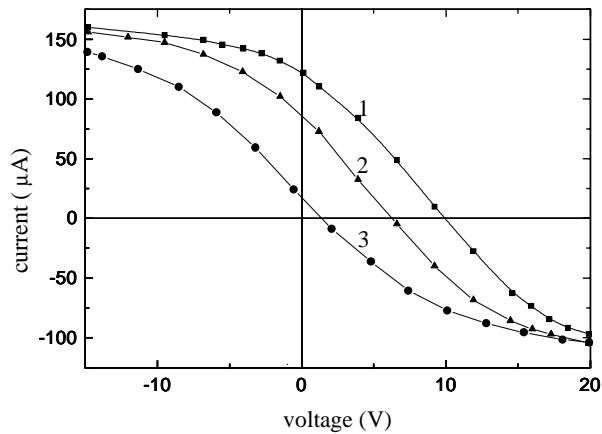

(7 b)

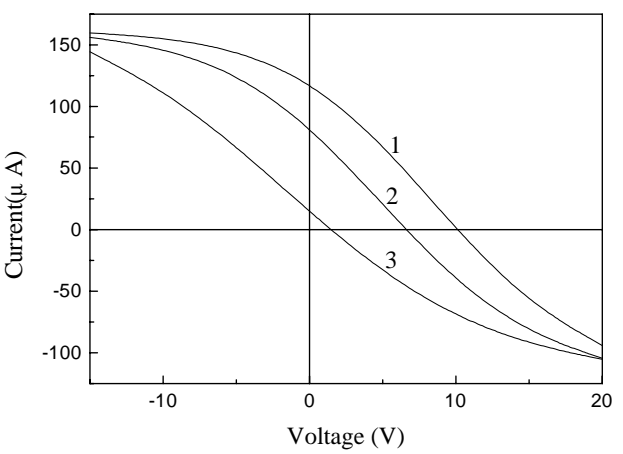

Figure 7. a) Experimental and b) theoretical characteristic of the double probe having frontal probe configuration. The distance between the probe was kept fixed at $0.51 \mathrm{~m}$. In this case the plasma parameters converge to: curve 1: $R_{1}=0.42, R_{2}=0.40, T_{e 1}=$ $5.3 \mathrm{eV}, T_{e 2}=4,3 \mathrm{eV}, N_{1}=1.85 \times 10^{17} \mathrm{~m}^{-3}, N_{2}=3.45 \times 10^{17}$ $\mathrm{m}^{-3}, \Delta V_{p}=4.3 \mathrm{~V}$. curve $2: R_{1}=0.52, R_{2}=0.50, T_{e 1}=5.4$ $\mathrm{eV}, T_{e 2}=4,4 \mathrm{eV}, N_{1}=1.85 \times 10^{17} \mathrm{~m}^{-3}, N_{2}=3.40 \times 10^{17}$ $\mathrm{m}^{-3}, \Delta V_{p}=1 \mathrm{~V}$. curve 3: $R_{1}=0.76, R_{2}=0.75, T_{e 1}=5.6 \mathrm{eV}$, $T_{e 2}=5.5 \mathrm{eV}, N_{1}=1.83 \times 10^{17} \mathrm{~m}^{-3}, N_{2}=3.40 \times 10^{17} \mathrm{~m}^{-3}$, $\Delta V_{p}=0.7 \mathrm{~V}$.

These plasma parameters obtained by the numerical fitting are consistent with the experimental data taken in a low mercury arc discharge, as depicted in Fig. 6. Note that the $R$ values at the probe positions are approximately equals according to experimental data [6]. In this reference, it is shown that the difference between each parameter $\Delta_{e}$ and $\Delta N$ is significant only for lower $R$-values. When the plasma becomes more uniform (curve 3 ), the floating potential of the two frontal probes is acceptable as being the difference of the plasma potential at the positions of the probes $\left(\Delta V_{P}\right)$, as already discussed in 2.1 .

Figure 8 (dashed line) shows the theoretical correction necessary to compute the difference of the two probes 
plasma potential, according to eq.(16). This result, when divided by the distance between the probes $(\mathrm{s}=0.51 \mathrm{~m})$ gives an estimation of the axial electric field along the plasma column. Fig. 9 shows clearly that the axial electric field is a decreasing function of the electron drift velocity. As $R_{1}$ and $R_{2}$ have almost equal values, we have taken the mean value of $R$ to draw the Figs. 8 and 9.

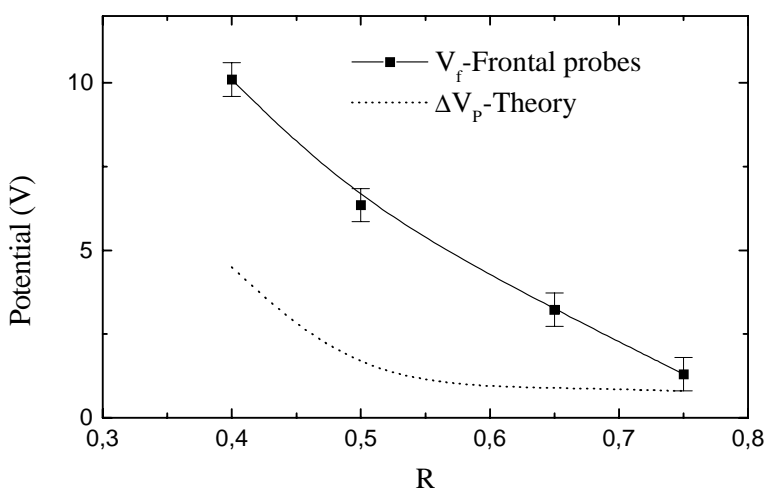

Figure 8. Effect of the electron drift velocity on the floating potential of the double probe. Experimental data(solid line) was taken from the figure 7 (a) and the dashed line indicates the plasma potential variation computed by equation (16) for $T_{e 1}>T_{e 2}$.

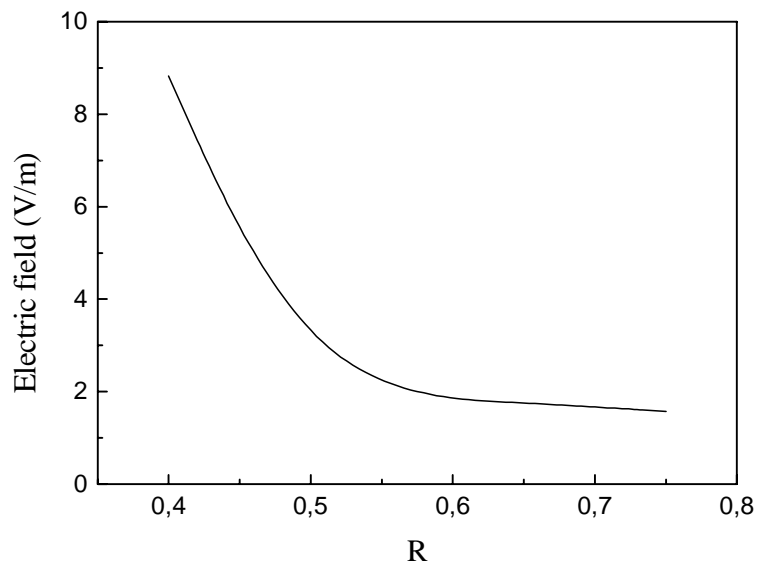

Figure 9. Axial electric field of the plasma column as a function of the electron drift velocity.

\section{Conclusion}

The directional double probe studies developed in this work consider a plasma in which electrons have a drifted maxwellian distribution. The distance between the probes is very large such that the plasma parameters could be different, where the probes are located. The magnitude of the drift is represented by he ratio of the electron drift velocity to thermal velocity $(R)$. A general equation for the double probe characteristic is deduced. For practical use of the double probe, we deduced analytical relations in which are shown the direct dependency of the floating potential and of the slope of the double probe characteristic upon the $R$ parameter.

Data from a plasma column of a low-pressure mercury arc discharge were used to proceed an interactive method in order to fit experimental characteristic curves with theoretical ones. The results show good agreement for the case of frontal probes configuration. This technique was especially applied to verify the effects of the electron drift velocity on the plasma potential and on the axial electric field along the positive column. As other applicability, depending on the nature of the gas, this theory could be used in various cold plasma reactors where non-uniform plasmas are usually produced. An application of this double probe technique could be the measurements of $\Delta T_{e}$ and $\Delta V_{P}$ in the different regions of a discharge as for example in the different plasmas formed in the $\mathrm{H}$ mode [7] generated in a inductively r.f plasma reactor. Also, this technique could be used to characterize quiescent plasmas generated in double plasma machines [8], where two different plasmas are separated by polarization grids. In this case, the $R$-values of the plasmas could be made different by acceleration of electrons and ions by means of the grids. This effect also occurs in plasma jet reactors [9] based in a glow discharge plasma column where a plasma beam is generated by a geometric constriction of the discharge tube. However, in most of these examples, the plasma parameters in the region between the probes, are non-uniform. Thus, a spatial profile of these parameters could be obtained by varying the distance between the probes.

\section{References}

[1] F.F. Chen,in Plasma Diagnostic Techniques (Academic, New York, 1965) p. 136.

[2] J.D. Swift, and Schar, in Electrical probes for plasma diagnostic (M.J.R. Iliffe Books Ltd, London, 1970) 137.

[3] H.S. Maciel, and J.E. Allen, J. Plasma Physics 42, 321 (1989).

[4] G. Petraconi, H.S. Maciel, and M. Uehara, Fourth Symposium on Double Layers and Other Nonlinear Potential Structures in Plasma, Innsbruch, Roman W. Schrittwieser Ed. 244 (1992).

[5] S. Ariga, Journal of the Physical Society of Japan, 31, 4, 1221(1971).

[6] G. Petraconi, et. al. To be published.

[7] J. Amorin, Journal Vac. Sci. Technology, B 9 02, 362 (1991).

[8] G. Hassal and J.E. Allen, J. Phys. D: Appl. Phys. 30, 381 (1997).

[9] W. L. Urruchi, H.S. Maciel, G. Petraconi, and C. Otani, Journal of Techincal Physics, 40, 1, 419 (1999). 